\title{
Aprovechamiento de Sistemas de Almacenamiento Energético Mediante Baterías para la Mitigación de Desequilibrios en Microrredes Eléctricas
}

\section{Use of Battery Energy Storage System to Mitigate Unbalances in Microgrids}

\author{
Danny Ochoa Correa ${ }^{1}$ https://orcid.org/0000-0001-5633-1480 \\ ${ }^{1}$ Universidad de Cuenca, Cuenca, Ecuador \\ danny.ochoac@ucuenca.edu.ec

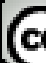

Esta obra está bajo una licencia internacional

Creative Commons Atribución-NoComercial 4.0.

Enviado:

$2021 / 01 / 07$

Aceptado:

$2021 / 02 / 04$

Publicado:

\section{Resumen}

La proliferación de cargas monofásicas en los sistemas de distribución eléctrica y su naturaleza asimétrica en su operación en tiempo real es la principal causa de desequilibrios de tensión y de corriente en microrredes eléctricas y en sistemas eléctricos débiles y aislados. Según la severidad del desequilibrio, esta condición podría comprometer el normal funcionamiento de los distintos componentes del sistema, reducir su eficiencia, perjudicar su vida útil y, por último, degradar la calidad del suministro eléctrico. En este artículo se propone una estrategia de control concebida para facultar a los convertidores electrónicos de potencia asociados a los sistemas de almacenamiento energético en baterías (BESS) la realización de labores de mitigación del desequilibrio introducido por la carga trifásica asimétrica en determinados puntos del sistema. La propuesta se basa en la teoría de las componentes simétricas instantáneas, que está diseñada para su implementación en los esquemas de control convencionales incorporados en la electrónica del BESS. La efectividad de la propuesta es evaluada a partir de un sistema eléctrico de pruebas implementado en MATLAB/Simulink. Los resultados obtenidos confirman el correcto desempeño de la estrategia de control y permiten verificar el cumplimiento de las hipótesis de investigación.

Palabras clave: microrred, sistema de almacenamiento energético en baterías, desequilibrio de tensión, componentes simétricas instantáneas, convertidor electrónico de potencia.

Abstract

The massive presence of single-phase loads in electrical distribution systems and their asymmetric nature in real-time operation is the leading cause of voltage and current unbalances in microgrids and weak isolated power systems. Depending on the severity of the unbalance, this condition might compromise the expected performance of system components, reducing efficiency, and

Sumario: 1 Introducción, 2 Desequilibrios en microrredes eléctricas y Método de detección, 3 Compensación de desequilibrios mediante BESS, 4 Resultados y Discusión, 5 Conclusiones, 6 Anexos.

Como citar: Ochoa, D. (2021). Aprovechamiento de Sistemas de Almacenamiento Energético Mediante Baterías para la Mitigación de Desequilibrios en Microrredes Eléctricas. Revista Tecnológica - Espol, 33(1), 18-34. http://www.rte.espol.edu.ec/index.php/tecnologica/article/view/798 
negatively impacting its lifetime plus the degradation of the power supply quality. This article proposes a control strategy designed to enable the electronic converters associated with battery energy storage systems (BESS) to mitigate the unbalance introduced by the asymmetrical three-phase load at specific points of the system. The proposal is based on the instantaneous symmetrical components' theory, and it is designed for implementation in conventional control schemes incorporated in BESS electronics. The effectiveness of the proposal is evaluated on a testbench implemented in MATLAB/Simulink. The simulation results confirm the control strategy's efficiency and validate the research hypotheses.

Keywords: microgrid, battery energy storage system, voltage unbalance, instantaneous symmetrical components, power electronic converter.

\section{Introducción}

La tendencia creciente de la demanda de energía experimentada por los sistemas eléctricos a nivel mundial ha propiciado que el nivel de cargabilidad de la infraestructura necesaria para producir, transportar y distribuir la electricidad a los consumidores se acerque cada vez a su límite superior. Con el objeto de evitar alcanzar este límite, los operadores de los sistemas suelen llevar a cabo proyectos de repotenciación y expansión del sistema eléctrico (en todas sus etapas), cuyos costos asociados suelen ser cubiertos con fondos provenientes de las arcas públicas y de las inversiones del sector privado (Agencia de Regulación y Control de Electricidad-ARCONEL, 2018a). A fin de aliviar la elevada carga económica que supone la expansión del sector de generación y de transmisión de electricidad, además de obtener otro tipo de réditos, en la última década ha cobrado un enorme interés el estudio y la implementación de microrredes eléctricas, cuyo grado actual de madurez tecnológica constituye una alternativa económicamente viable (G. V. B. Kumar \& Palanisamy, 2020). Por definición, una microrred es un grupo de cargas interconectadas y recursos energéticos distribuidos que actúan como un solo ente controlado dentro un sistema eléctrico interconectado (red principal), el cual posee una topología claramente delimitada. La microrred puede ser conectada o desconectada de la red principal de tal manera que esta pueda operar tanto en modo conectado a la red como en modo aislado (Gundumalla \& Eswararao, 2018). En la arquitectura de una microrred, las unidades de generación pueden localizarse más cerca de los consumidores (a nivel del sistema de distribución), tener un tamaño sensiblemente inferior que las grandes centrales convencionales y facilitar la diversificación de su fuente energética primaria (renovable no convencional, en su mayoría), de tal forma que la energía producida fluya desde los generadores hacia las cargas, sin tener que pasar por el sistema de transmisión, lo que reduce tanto las pérdidas energéticas como el nivel de congestión de la infraestructura. Este paradigma de generación recibe el nombre de "generación distribuida", cuyos beneficios ambientales, gracias a la reducción en la quema de combustibles fósiles, y técnicos, por la reducción de pérdidas y el aumento en la continuidad del suministro eléctrico, han sido discutidos ampliamente en la literatura de este campo disciplinar (Karimi et al., 2021; T. P. Kumar et al., 2015; Saponara et al., 2019). En muchos casos, las microrredes suelen incorporar sistemas de almacenamiento energético diferentes para garantizar la continuidad del suministro y brindar ciertos servicios de soporte a la red, como el control de tensión y de frecuencia, en particular, cuando esta última se encuentra operando en modo aislado (G. V. B. Kumar \& Palanisamy, 2020). En la Figura 1 se muestra una configuración típica de una microrred eléctrica (las flechas en azul representan los flujos de potencia en los distintos elementos del sistema).

En el contexto del sistema eléctrico ecuatoriano existen ciertas redes que, debido a sus particularidades, pueden ser consideradas microrredes eléctricas, sin que estas hayan sido necesariamente concebidas como tal en sus inicios. Entre los ejemplos más destacables se encuentran los sistemas aislados de las islas Galápagos (San Cristóbal, Baltra-Santa Cruz y Floreana) y los proyectos de electrificación rural en sectores remotos de la región amazónica, los cuales, dada la imposibilidad técnica-económica de interconectarlos al sistema nacional de transmisión, han tenido que autogestionarse energéticamente a fin de garantizar la calidad y continuidad del suministro a los distintos tipos de consumidor. Más allá de las múltiples ventajas que presenta la arquitectura de una microrred eléctrica, desde un punto de vista técnico, ambiental y económico, existen también desafíos de diversa índole que han despertado el interés de la comunidad científica (G. V. B. Kumar \& Palanisamy, 2020; Nájera et al., 2019). Uno de ellos es el problema relacionado con el desequilibrio de tensiones y corrientes, materia que es objeto del estudio presentado en este trabajo. 
El desequilibrio o desbalance de tensiones se presenta en sistemas trifásicos cuando el módulo y el ángulo de las tensiones entre fases consecutivas no son iguales (Nájera et al., 2019). La principal causa de los desequilibrios de tensión en un sistema de distribución suele ser el consumo de potencia asimétrico por parte de las cargas trifásicas, lo que propicia la circulación de corrientes desequilibradas. La naturaleza de este consumo de potencia se debe a la proliferación de cargas monofásicas, cuyos perfiles de consumo individuales, vistos en tiempo real, difieren en cada una de las fases. El efecto del desequilibrio es mucho más notorio en redes débiles y aisladas, debido a su elevado valor de impedancia en el punto de conexión de la carga trifásica (Etxegarai et al., 2015). El desequilibrio de tensión afecta de manera directa a la calidad de la energía suministrada a los consumidores (industrial, comercial, residencial y otros), y puede impactar de forma negativa al funcionamiento de cargas trifásicas, como motores, hornos eléctricos, variadores de frecuencia y sistemas de carga de vehículos eléctricos, entre otros; esto provoca la reducción de la eficiencia y la vida útil de estos artefactos (Adouni \& J. Marques Cardoso, 2021; Antić et al., 2021). Consecuencias similares podría experimentar el equipamiento eléctrico instalado aguas arriba de la carga (generadores y transformadores) si son sometidos a elevados niveles de desequilibrio (de tensión y/o corriente) de forma sostenida en el tiempo. Es tal la importancia de este indicador, que el código de red ecuatoriano incluye en la regulación ARCONEL 005/18 los valores máximos admisibles de desequilibrio que deberá presentar el sistema de distribución (en sus distintos niveles) para brindar un servicio eléctrico con adecuados márgenes de calidad (Agencia de Regulación y Control de Electricidad-ARCONEL, 2018b).

\section{Figura 1}

Configuración típica de una microrred eléctrica

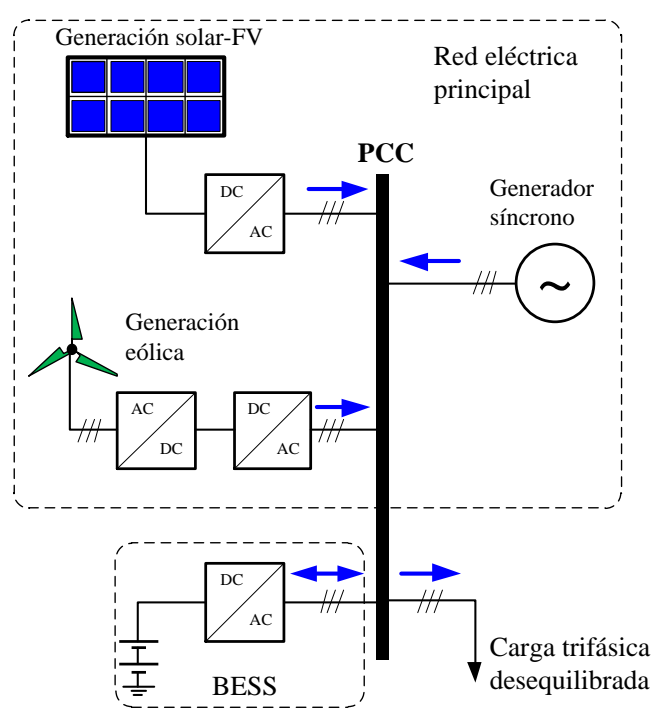

Fuente: Elaboración propia

Si bien los operadores de este tipo de redes han invertido enormes esfuerzos por mantener las redes trifásicas relativamente equilibradas, por ejemplo, mediante criterios de reparto equitativo de la potencia instalada en cada una de las fases durante la etapa de diseño, es el comportamiento real de las cargas el que hace que el desequilibrio sea un problema latente cuya mitigación deba alcanzarse mediante la intervención de agentes energéticos exógenos a la propia carga trifásica. Pues bien, apelando a la flexibilidad operativa que presentan las interfaces de conexión a red basadas en el uso de convertidores electrónicos de potencia y considerando que su participación en los sistemas eléctricos de potencia hoy en día es altamente significativa, en este trabajo se plantea la utilización de un sistema de almacenamiento energético en baterías (BESS, por sus siglas en inglés) como medida efectiva de mitigación de desequilibrios.

En concreto, en este manuscrito se propone una estrategia de control para ser implementada en el convertidor electrónico de potencia asociado a un sistema BESS, de tal forma que faculte a este agente energético a inyectar corrientes trifásicas en el punto de conexión común (PCC), con miras a 
compensar la corriente desequilibrada de la carga y permitir que esta última sea percibida como equilibrada por el resto de los componentes eléctricos situados aguas arriba del PCC. Para tal efecto, en el desarrollo analítico conducente a la generación de las señales de control a ser aplicadas al convertidor electrónico del BESS, se toma como base metodológica el concepto de las componentes simétricas instantáneas presentado por Ghosh y Joshi (2000). Como resultado, se obtienen expresiones que permiten evaluar en tiempo real las señales de consigna de corriente, que deberán ser inyectadas por el convertidor del BESS en el PCC para mitigar el desequilibrio. Estas expresiones, formuladas en términos de las variables eléctricas que pueden ser medidas en bornes del convertidor, toman en consideración el factor de potencia y se deducen a partir de un modelo de carga trifásica desequilibrada conectada en estrella. El desempeño de la estrategia de control propuesta se evalúa sobre un banco de pruebas diseñado para los intereses científicos de este trabajo e implementado en el entorno de simulación MATLAB/Simulink.

\section{Desequilibrios en microrredes eléctricas y Método de detección Definición de desequilibrio, problemática y estándares}

El desequilibrio o desbalance (de tensión y/o corriente) se presenta en sistemas trifásicos cuando el módulo y el ángulo de las tensiones y/o corrientes entre fases consecutivas no son iguales. Exceptuando las fallas eventuales que pueden ocurrir en un sistema, la principal causa de los desequilibrios de tensión suele ser el consumo de potencia asimétrico por parte de las cargas, lo que se traduce en la aparición de corrientes desequilibradas. Si se analizan los distintos tipos de consumidores en un sistema, el sector residencial presenta un gran número de acometidas monofásicas, cuyos hábitos de consumo individuales son fuente de constantes desequilibrios. En lo que respecta a las instalaciones industriales y comerciales, estas pueden presentar un consumo eléctrico muy equilibrado a nivel de diseño. No obstante, en su operación en tiempo real es muy probable que se detecten niveles de desequilibrio producidos dentro de la misma instalación debido, quizá, a una mala distribución de los equipamientos, sobrecarga en alguna de las fases o conexiones con una alta impedancia causadas por malos contactos entre componentes. La Figura 2 muestra el perfil de consumo eléctrico real de una acometida trifásica en el sistema de distribución ecuatoriano (hospital público, localizado en la provincia de Morona Santiago). Este registro, obtenido con un elevado grado de resolución temporal, pone en evidencia que, a pesar de los esfuerzos realizados durante la planificación de la expansión del sistema eléctrico para mantener las redes trifásicas sensiblemente equilibradas (por medio de políticas y criterios de diseño), es el comportamiento real de las cargas lo que hace que el desequilibrio sea un problema latente, cuya mitigación deberá conseguirse mediante la intervención de agentes energéticos externos, implementando en sus esquemas de control ciertas técnicas de compensación.

El desequilibrio de tensión afecta de manera directa a la calidad de la energía suministrada a los consumidores, independientemente del nivel que estos ocupen en el sistema (industrial, comercial, residencial y otros), por lo que el impacto dependerá del uso final de la energía. Es así como, en el caso de las cargas trifásicas, tales como motores, hornos eléctricos y variadores de frecuencia, entre otras, la operación bajo un régimen continuado de desequilibrio puede repercutir en su funcionamiento normal y sufrir daños constructivos, y, en consecuencia, se produzca la reducción de la eficiencia y vida útil de los artefactos. Consecuencias similares podrían experimentar otros elementos del sistema, como los generadores y transformadores, si estos son sometidos a condiciones operativas extremas de desequilibrio.

En la actualidad, entre los estándares más extendidos para evaluar el grado de desequilibrio en una red de distribución, están el IEEE 112-x y el IEC 1000-3-x. En este estudio, se tomará como referencia el segundo estándar, por cuanto este ha sido adoptado por la normativa ecuatoriana ARCONEL 005/18 para definir los índices de calidad del servicio de distribución y comercialización de energía eléctrica (Agencia de Regulación y Control de Electricidad-ARCONEL, 2018b). En este se define el factor de desequilibrio de tensión (VUF, por sus siglas en inglés), expresado en (1), en donde V1 y V2 son las componentes simétricas positiva y negativa de la tensión trifásica evaluada, respectivamente. El valor máximo permitido para los niveles de media y baja tensión corresponde al 2 $\%$. 
$\mathrm{VUF}=\left|\frac{V_{2}}{V_{1}}\right| \times 100 \%$

Figura 2

Perfil de consumo eléctrico registrado en una acometida trifásica: a) potencia activa; b) tensión fase-neutro; c) corriente y d) desequilibrio de tensión de acuerdo con ARCONEL 005/18

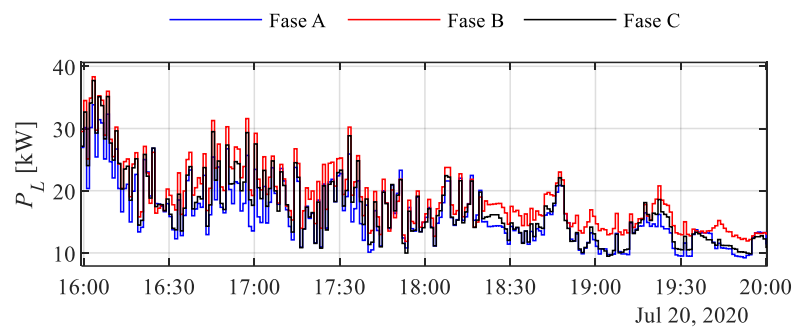

(a)

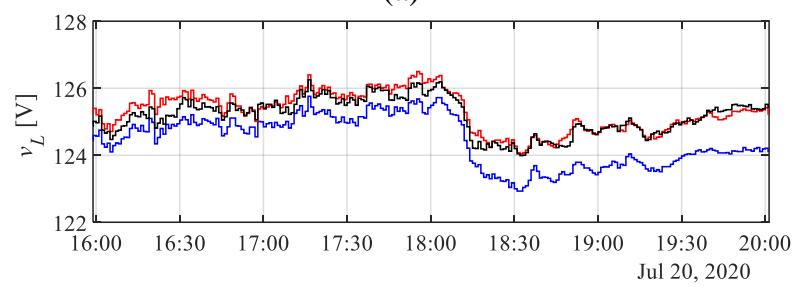

(b)

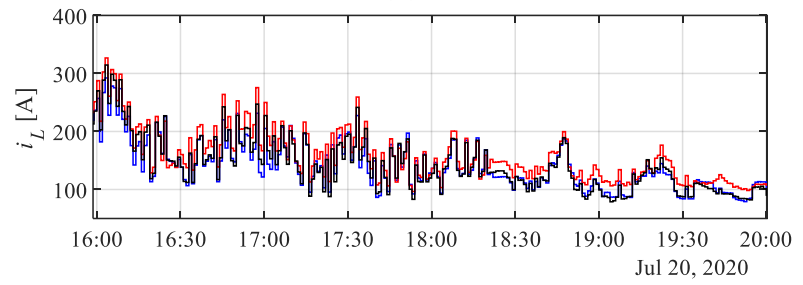

(c)

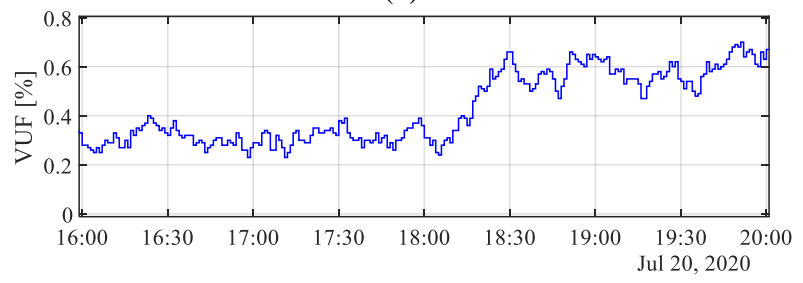

(d)

\section{Componentes simétricas instantáneas}

En la teoría clásica de los sistemas eléctricos de potencia, los sistemas desequilibrados son estudiados mediante la aplicación del concepto de las componentes simétricas (o de secuencia) propuestas por C. L. Fortescue (1918). El empleo de esta conveniente herramienta analítica ha permitido reducir, de forma considerable, el tratamiento analítico de redes que experimentan distintos tipos de desequilibrios, sean estos producidos por condiciones de falla o por la naturaleza de ciertos parámetros de la red operando en condiciones normales (Grainger \& Stevenson, 2004; Kundur, 1994). La aplicación del método de las componentes simétricas a una señal trifásica de tensión y/o corriente arroja como resultado una terna de fasores de secuencia: positiva, negativa y cero, en donde la presencia de estos dos últimos indica la operación desequilibrada del sistema. No obstante, en aplicaciones en tiempo real, que es el objeto de este estudio, el empleo de un análisis fasorial podría restar efectividad a las labores de monitorización y compensación, ya que este ha sido concebido para ser aplicado en señales eléctricas con frecuencia constante y operación en estado estacionario. En su lugar, es preferible registrar la variación "instantánea" de las variables eléctricas (tensión y/o corriente) para conseguir señales de compensación más fiables. Para ello, en Ghosh y Joshi (2000) se presenta el concepto de las componentes simétricas instantáneas, el cual establece que un conjunto de tensiones trifásicas instantáneas $v_{a}(t), v_{b}(t)$ y $v_{c}(t)$ puede ser descompuesto como sigue: 


$$
\left[\begin{array}{c}
v_{a 0} \\
v_{a 1} \\
v_{a 2}
\end{array}\right]=\frac{1}{\sqrt{3}}\left[\begin{array}{ccc}
1 & 1 & 1 \\
1 & a & a^{2} \\
1 & a^{2} & a
\end{array}\right]\left[\begin{array}{c}
v_{a} \\
v_{b} \\
v_{c}
\end{array}\right]
$$

En donde $v_{a 0}, v_{a 1}$ y $v_{a 2}$ son las componentes simétricas instantáneas (referida a la fase a) de la tensión trifásica estudiada: cero, positiva y negativa, respectivamente. Dado que $a=1 e^{j 120^{\circ}}$, los vectores instantáneos $v_{a 1}$ y $v_{a 2}$ son complejos conjugados entre sí y $v_{a 0}$ es una cantidad real. Por tanto, para representar gráficamente las componentes de secuencia obtenidas, resultará útil emplear el plano complejo. En este punto, es preciso indicar que la ecuación (2) es válida también para la descomposición de corrientes trifásicas instantáneas.

Para ilustrar el método considérese la señal trifásica de tensión desequilibrada, expresada en la parte central de la Figura 3. Cada vez que se toma una muestra de las tensiones $v_{a}, v_{b}, v_{c}$, se evalúa (2) con el fin de obtener una terna de valores que representen a las componentes $v_{a 0}$, $v_{a 1}$ y $v_{a 2}$ para ese instante de tiempo. Conforme se avanza en el barrido de la señal trifásica, se va trazando en el plano complejo los valores adoptados por $v_{a 1}$ y $v_{a 2}$, como se ilustra en la Figura 3. Nótese que el lugar geométrico descrito por $v_{a 1}$ es el reflejo de aquel producido por $v_{a 2}$. La falta de correlación entre las dos curvas denota el desequilibrio de la señal trifásica estudiada, siendo esta más acentuada mientras más severo sea el desequilibrio. Por tanto, es de esperar que, en caso de tener un sistema equilibrado, los lugares geométricos de $v_{a 1}$ y $v_{a 2}$ se solapen perfectamente. He aquí la utilidad de emplear este concepto para monitorizar el desequilibrio de tensión y/o corriente y para generar las señales de compensación correspondientes. En el ejemplo mostrado en la Figura 3, se han barrido las señales de tensión trifásicas (de $60 \mathrm{~Hz}$ ) en una ventana temporal de dos ciclos y un tiempo de muestreo de $0.1 \mathrm{~ms}$.

Figura 3

Detección de desequilibrios mediante el concepto de las componentes simétricas instantáneas

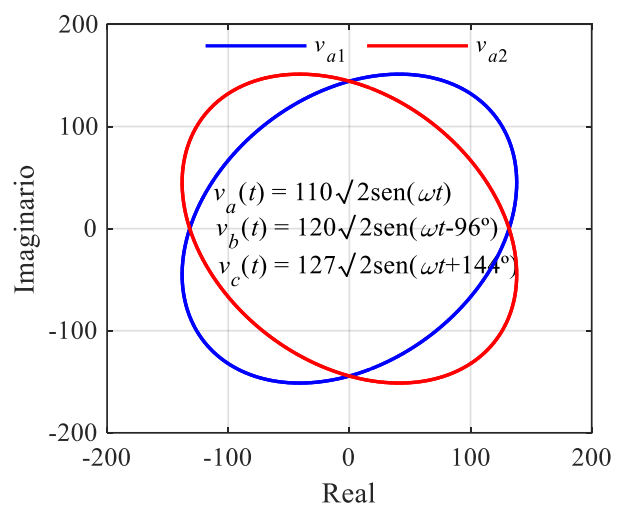

Fuente: Elaboración propia

\section{Compensación de desequilibrios mediante BESS}

\section{Propuesta metodológica}

La Figura 4 muestra la representación simplificada de una microrred eléctrica alimentando a una carga trifásica desequilibrada, en cuyo punto de conexión común a la red (PCC) se encuentra un sistema de almacenamiento energético mediante baterías (BESS) con interfaz de conexión basada en convertidor electrónico de potencia (CEP), similar a la topología de la microrred ilustrada en la Figura 1. Con el objeto de exponer con claridad el planteamiento de la metodología propuesta, la red eléctrica situada aguas arriba del PCC es representada por su equivalente Thévenin (Figura 4). En condiciones normales de operación, las potencias activas y reactivas demandadas por cada una de las fases de la carga son suplidas en mayor medida por la red eléctrica principal y, de forma complementaria, por el BESS. Independientemente de si el BESS inyecta o absorbe potencia del PCC, las corrientes trifásicas generadas por el CEP serán siempre equilibradas, por tanto, el desequilibrio de corrientes y tensiones ocasionado por la carga es asumido en su totalidad por la red eléctrica principal. Esta naturaleza desequilibrada y cambiante de la carga, que se traduce en la aparición de potencias instantáneas 
pulsantes, circulación de corriente por el neutro, desequilibrio de tensión en bornes, podría generar efectos adversos en los elementos que constituyen la red situada aguas arriba del PCC (generadores y transformadores, principalmente) y en la propia carga, como ya se expuso en la sección introductoria.

La metodología que se propone en este artículo parte de la premisa de que, si no es posible cambiar la naturaleza desequilibrada de la carga en su operación en tiempo real, lo que se puede hacer es aprovechar el BESS conectado en el PCC para que este inyecte corrientes trifásicas que permitan compensar el desequilibrio "aguas arriba", de tal manera que el resto de los componentes de la microrred perciba a la carga como equilibrada, con los consiguientes beneficios operativos. Se propone, entonces, un ajuste en la filosofía de control del CEP asociado al BESS para que este continúe desempeñando su rol dentro de la microrred eléctrica, al mismo tiempo que sea empleado para desempeñar las labores de mitigación del desequilibrio introducido por la carga.

Figura 4

Representación simplificada de una microrred eléctrica

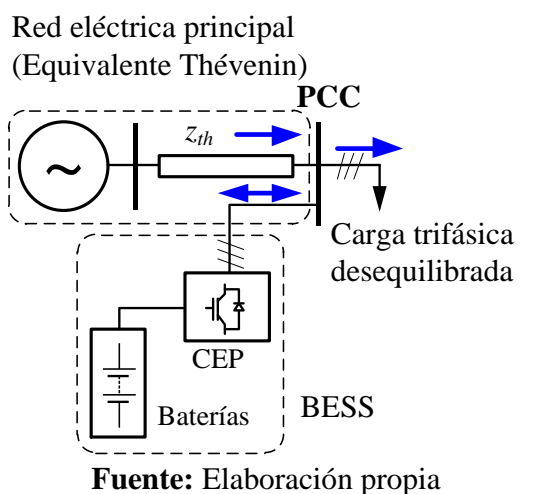

Para el planteamiento de la propuesta, se acudirá a lo expresado en la Figura 5, que muestra la representación trifásica de la microrred de la Figura 4. En esta se asume que la única fuente de desequilibrio proviene de la carga (conectada en estrella), por tanto, la tensión trifásica de la red eléctrica situada aguas arriba del PCC posee la forma expresada en (3), donde $V_{m}$ es la amplitud máxima y $\omega$ es la frecuencia angular de la red. Tanto la tensión fase-neutro en el PCC como la corriente consumida por la carga en cada fase están denotadas por $v_{L}$ y $i_{L}$, respectivamente. El CEP, que para este tipo de aplicaciones opera en modo convertidor en fuente de corriente (CSC, por sus siglas en inglés), es representado por medio de una fuente controlada de corriente que inyecta una señal trifásica $i_{B E S S}$, cuyo cálculo constituye el objeto principal de este trabajo. Esta simplificación está sustentada en los resultados publicados en Huang et al. (2012); Li et al., (2018) y Radwan et al. (2020), en donde se verifica que el CEP adopta un comportamiento equivalente al de una fuente de corriente desde la perspectiva de la red en el PCC, en el cumplimiento de las señales de consigna de potencia y activa establecidas en su sistema de control.

Figura 5

Representación trifásica de la microrred eléctrica

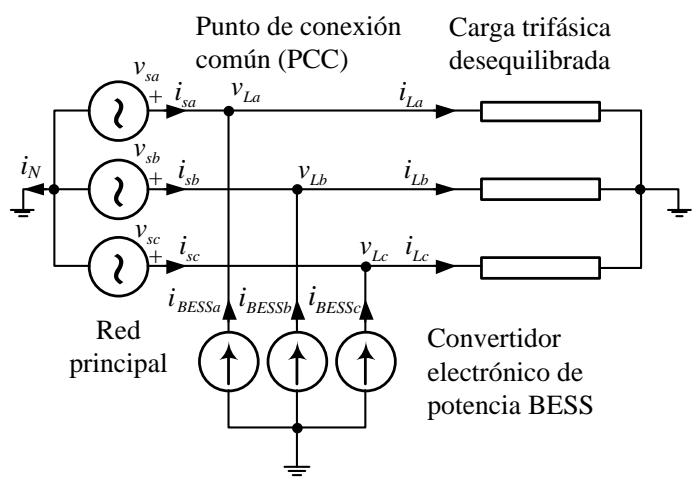

Fuente: Elaboración propia 


$$
\begin{gathered}
v_{s a}=V_{m} \operatorname{sen}(\omega t) \\
v_{s b}=V_{m} \operatorname{sen}\left(\omega t-120^{\circ}\right) \\
v_{s c}=V_{m} \operatorname{sen}\left(\omega t+120^{\circ}\right)
\end{gathered}
$$

Dado que el desequilibrio de tensión/corriente en el sistema es ocasionado por la asimetría de la carga (en términos de la potencia activa/reactiva consumida en cada fase), se partirá del concepto de las componentes simétricas instantáneas descrito en la sección II-B para determinar una función de control. La idea consiste en encontrar una relación entre las corrientes y las tensiones de carga, así como una acción de control que permita incrementar la correlación (en tiempo real) del lugar geométrico de las componentes positiva y negativa de la corriente inyectada por la red en el PCC (como se ilustró en la Figura 3, con la tensión), y, con esto, reducir el grado de desequilibrio visto desde la red principal. Del diagrama de la Figura 5 se infiere que la red eléctrica principal habrá alcanzado la condición de equilibrio cuando:

$$
\underbrace{i_{s a}+i_{s b}+i_{s c}}_{\substack{\text { Corriente circulante } \\ \text { por el neutro }}}=0
$$

La expresión (4) constituye el primer objetivo de control que se busca con la implementación de la propuesta. A continuación, haciendo uso de la ecuación (2), se deduce que la componente de secuencia positiva de la tensión fase-neutro instantánea de la red principal, $v_{s a 1}$, posee la forma:

$$
v_{s a 1}=\frac{1}{\sqrt{3}}\left(v_{s a}-\frac{1}{2} v_{s b}-\frac{1}{2} v_{s c}\right)+j\left(\frac{1}{2} v_{s b}-\frac{1}{2} v_{s c}\right)
$$

Debido a que $v_{s a 2}$ es el complejo conjugado de $v_{s a 1}$, su deducción se omite en este punto. Luego, operando sobre (5), se obtiene el argumento del vector complejo, $v_{s a 1}$ :

$$
\angle\left(v_{s a 1}\right)=\tan ^{-1}\left\{\frac{\frac{\sqrt{3}}{2}\left(v_{s b}-v_{s c}\right)}{v_{s a}-\frac{1}{2} v_{s b}-\frac{1}{2} v_{s c}}\right\}=\tan ^{-1}\left(\frac{k_{1}}{k_{2}}\right)
$$

Repitiendo el proceso analítico empleado en la deducción de las expresiones (5) y (6), y considerando que, para este caso, se utilizan las corrientes trifásicas inyectadas por la red principal en el PCC, se obtiene:

$$
\angle\left(i_{s a 1}\right)=\tan ^{-1}\left\{\frac{\frac{\sqrt{3}}{2}\left(i_{s b}-i_{s c}\right)}{i_{s a}-\frac{1}{2} i_{s b}-\frac{1}{2} i_{s c}}\right\}=\tan ^{-1}\left(\frac{k_{3}}{k_{4}}\right)
$$

Ahora bien, los argumentos de $v_{s a 1}$ y de $i_{s a 1}$ se pueden relacionar entre sí, como se indica en (8), en donde $\varphi$ es el ángulo del factor de potencia.

$$
\angle\left(v_{s a 1}\right)=\angle\left(i_{s a 1}\right)+\varphi
$$

Sustituyendo (6) y (7) en (8): 


$$
\tan ^{-1}\left(\frac{k_{1}}{k_{2}}\right)=\tan ^{-1}\left(\frac{k_{3}}{k_{4}}\right)+\varphi
$$

Aplicando la identidad trigonométrica $\tan (A+B)=(\tan A+\tan B) /(1-\tan A \tan B)$ en la expresión anterior, se obtiene:

$$
\frac{k_{1}}{k_{2}}=\frac{k_{3} / k_{4}+\tan \varphi}{1-\left(k_{3} / k_{4}\right) \tan \varphi}
$$

Para facilitar el manejo analítico de la expresión (10), cuando se sustituyan los valores $k_{1}-k_{4}$, definidos en (6) y (7), se introduce el factor $k=\tan \varphi / \sqrt{ } 3$. Luego de hacer esta sustitución y de reorganizar términos, se obtiene la expresión:

$$
\begin{aligned}
& \left(v_{s b}-v_{s c}-3 k v_{s a}\right) i_{s a}+\left(v_{s c}-v_{s a}-3 k v_{s b}\right) i_{s b}+ \\
& \left(v_{s a}-v_{s b}-3 k v_{s c}\right) i_{s c}=0
\end{aligned}
$$

En este punto, se pide al lector tener en cuenta la naturaleza ondulatoria que adquiere la potencia instantánea cuando el sistema opera bajo un régimen trifásico desequilibrado. En tal situación, la potencia total consumida por la carga en el dominio del tiempo poseerá dos componentes: una ondulatoria y una continua, siendo esta última la potencia promedio, $\overline{P_{L}}$ (como adelanto, se pide al lector observar la Figura 13(a)). En condiciones normales, esta potencia total ondulatoria es suplida, de forma mayoritaria, por la red eléctrica principal. Si bien, se espera que las acciones correctivas del CEP permitan obtener en el PCC una carga equivalente equilibrada (vista desde la red principal), no hay que descuidar que la carga conectada seguirá estando desequilibrada y, por tanto, esta seguirá demandando una potencia instantánea con componente ondulatoria. Aquí, se puede sacar provecho a la maleabilidad ofrecida por el CEP en relación con el control de potencia, al forzar a que este dispositivo asuma la componente ondulatoria de la potencia consumida por la carga y dejar que sea la red principal quien suministre la potencia promedio restante, $\overline{P_{L}}$ (Figura 13(b)). Este criterio de diseño, que constituye el segundo objetivo de control, puede formularse como sigue:

$$
\underbrace{v_{s b} i_{s a}+v_{s b} i_{s b}+v_{s c} i_{s c}}_{\substack{\text { Potencia instantanea suministrada } \\ \text { por la red principal }}}=\overline{P_{L}}
$$

A continuación se presentan las ecuaciones (4), (11) y (12) de forma matricial:

$$
\left[\begin{array}{ccc}
1 & 1 & 1 \\
\left(v_{s b}-v_{s c}-3 k v_{s a}\right) & \left(v_{s c}-v_{s a}-3 k v_{s b}\right) & \left(v_{s a}-v_{s b}-3 k v_{s c}\right) \\
v_{s a} & v_{s b} & v_{s c}
\end{array}\right]\left[\begin{array}{c}
i_{s a} \\
i_{s b} \\
i_{s c}
\end{array}\right]=\left[\begin{array}{c}
0 \\
0 \\
P_{L}
\end{array}\right]
$$

Teniendo en cuenta que para cada una de las fases del PCC se cumple que $i_{s}=i_{L}-i_{B E S S}$ y que $v_{s}=v_{L}$ (ver Figura 5), la aplicación del álgebra matricial en (13) da como resultado las ecuaciones (14)(16), las cuales son las señales de referencia trifásica que tienen que ser aplicadas al sistema de control del CEP. Se hace notar que las variables que constituyen este conjunto de ecuaciones pueden ser medidas en el PCC por parte del CEP, lo que facilita su implementación práctica. Además, con el ajuste del factor $k$ (que es proporcional a $\tan \varphi$ ) es posible controlar el factor de potencia en el PCC.

$$
i_{B E S S a}=i_{L a}-\frac{v_{L a}+\left(v_{L b}-v_{L c}\right) k}{v_{L a}^{2}+v_{L b}^{2}+v_{L c}^{2}} \overline{P_{L}}
$$




$$
\begin{aligned}
& i_{B E S S b}=i_{L b}-\frac{v_{L b}+\left(v_{L c}-v_{L a}\right) k}{v_{L a}^{2}+v_{L b}^{2}+v_{L c}^{2}} \overline{P_{L}} \\
& i_{B E S S c}=i_{L c}-\frac{v_{L c}+\left(v_{L a}-v_{L b}\right) k}{v_{L a}^{2}+v_{L b}^{2}+v_{L c}^{2}} \overline{P_{L}}
\end{aligned}
$$

\section{Caso de estudio}

Con el objeto de ilustrar la implementación de la propuesta planteada en la sección anterior y de evaluar la efectividad de las acciones de control brindadas por el CEP del BESS para la mitigación del desequilibrio en tiempo real, en este apartado se presenta un caso de estudio realizado sobre un sistema de pruebas diseñado para el efecto (Figura 6). El sistema consiste en una microrred de $220 \mathrm{~V}$ (valor eficaz de la tensión de línea) a $60 \mathrm{~Hz}$ alimentando a una carga trifásica y con sistema de almacenamiento energético en baterías (BESS). Al ser una microrred débil, el valor de la impedancia del modelo equivalente Thévenin está en el orden de 16 pu (valor calculado para una potencia base de 1MVA). La fuente de desequilibrio es la carga trifásica, la cual es representada por un modelo serie caracterizado por los siguientes parámetros: $Z_{L a}=10 e^{j 0^{\circ}} \Omega$ (fase a), $Z_{L b}=15 e^{j-50^{\circ}} \Omega$ (fase b) y $Z_{L c}=10 e$ ${ }^{j 30^{\circ}} \Omega$ (fase c), invariables en el tiempo (carga desequilibrada constante). En este estudio, se han dimensionado estas impedancias de tal manera que se introduzca un nivel de desequilibrio de tensión que exceda el valor máximo establecido estándar de referencia ( $2 \%$ ). Es preciso indicar que, de acuerdo con el enfoque utilizado en este trabajo, las corrientes trifásicas inyectadas por el CEP en el PCC provienen de una fuente controlada de corriente ideal, y, por tanto, se asume que esta dispone de un sustento energético ilimitado proporcionado, por ejemplo, por un banco de baterías. Esta asunción es válida para intervalos de análisis de corto plazo (en el orden de los pocos minutos), en donde el estado de carga típico de un BESS se percibe prácticamente constante (Ko \& Choi, 2021).

\section{Figura 6}

\section{Microrred eléctrica en estudio}

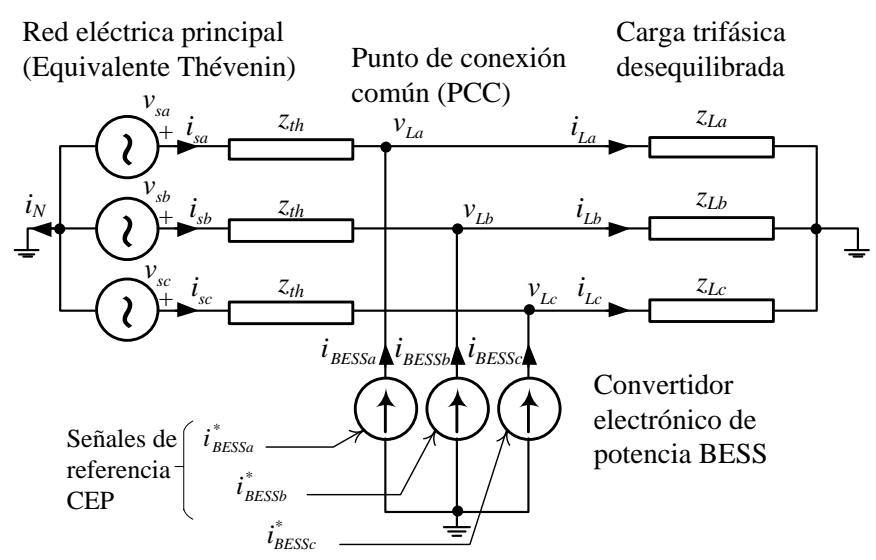

Fuente: Elaboración propia

En la Figura 7 se ofrece al lector un ejemplo de implementación de la estrategia de control propuesta. Este esquema simplificado ilustra el proceso de cálculo, en tiempo real, de las señales de referencia de corriente trifásica del CEP del BESS necesarias para la mitigación de los desequilibrios producidos por la carga. Este proceso inicia con la medición de los valores instantáneos de tensión trifásica en el PCC y de corriente trifásica de la carga, $v_{L}$ e $i_{L}$, respectivamente (en este ejemplo, se ha considerado un tiempo de muestreo $T_{\mathrm{s}}=0.1 \mathrm{~ms}$ ). Con las lecturas realizadas se calcula la potencia activa instantánea consumida por la carga y su valor promedio para luego evaluar las ecuaciones (14)-(16), deducidas en el apartado 3.1. Debido a que en este trabajo se ha empleado un modelo idealizado del sistema integral del BESS (fuente controlada de corriente), es preciso incluir una función de retraso (típicamente, de primer orden con constante de tiempo $T_{C}$ ) a fin de representar el tiempo de actuación del convertidor en el intervalo mientras que recibe la señal de consigna de corriente, $i^{*}{ }_{B E S S}$, hasta que esta es finalmente medida en bornes del CEP, $i_{B E S S}$. 
Figura 7

Esquema de implementación del controlador en el CEP del BESS

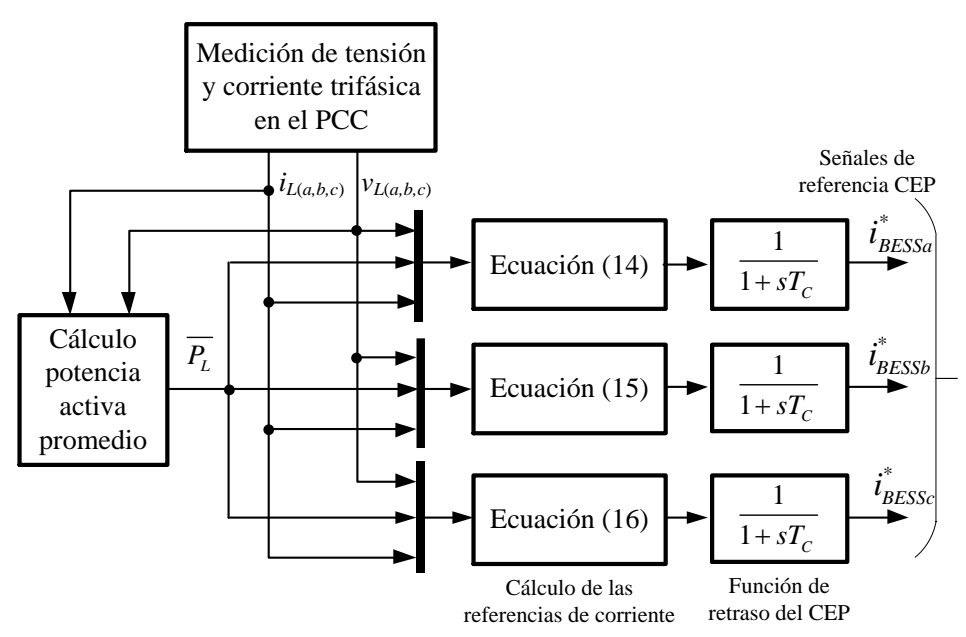

Fuente: Elaboración propia

\section{Resultados y Discusión}

La Figura 8 muestra la implementación del sistema de pruebas (Figura 6) en el entorno de simulación MATLAB/Simulink. En este modelo es posible también apreciar la implementación del esquema de control propuesto (Figura 7), el cual estará habilitado o no según las necesidades del estudio. Para ello, se utilizan los modelos integrados por defecto en la librería Simscape del simulador, con el objeto de representar los distintos elementos eléctricos que componen el sistema de pruebas y la metrología eléctrica necesaria para el registro de los datos, con el detalle que se muestra en la Figura 8. Los valores numéricos asignados a los distintos parámetros del modelo en la simulación se ofrecen en el Apéndice.

\section{Figura 8}

Implementación del sistema de pruebas en MATLAB/Simulink

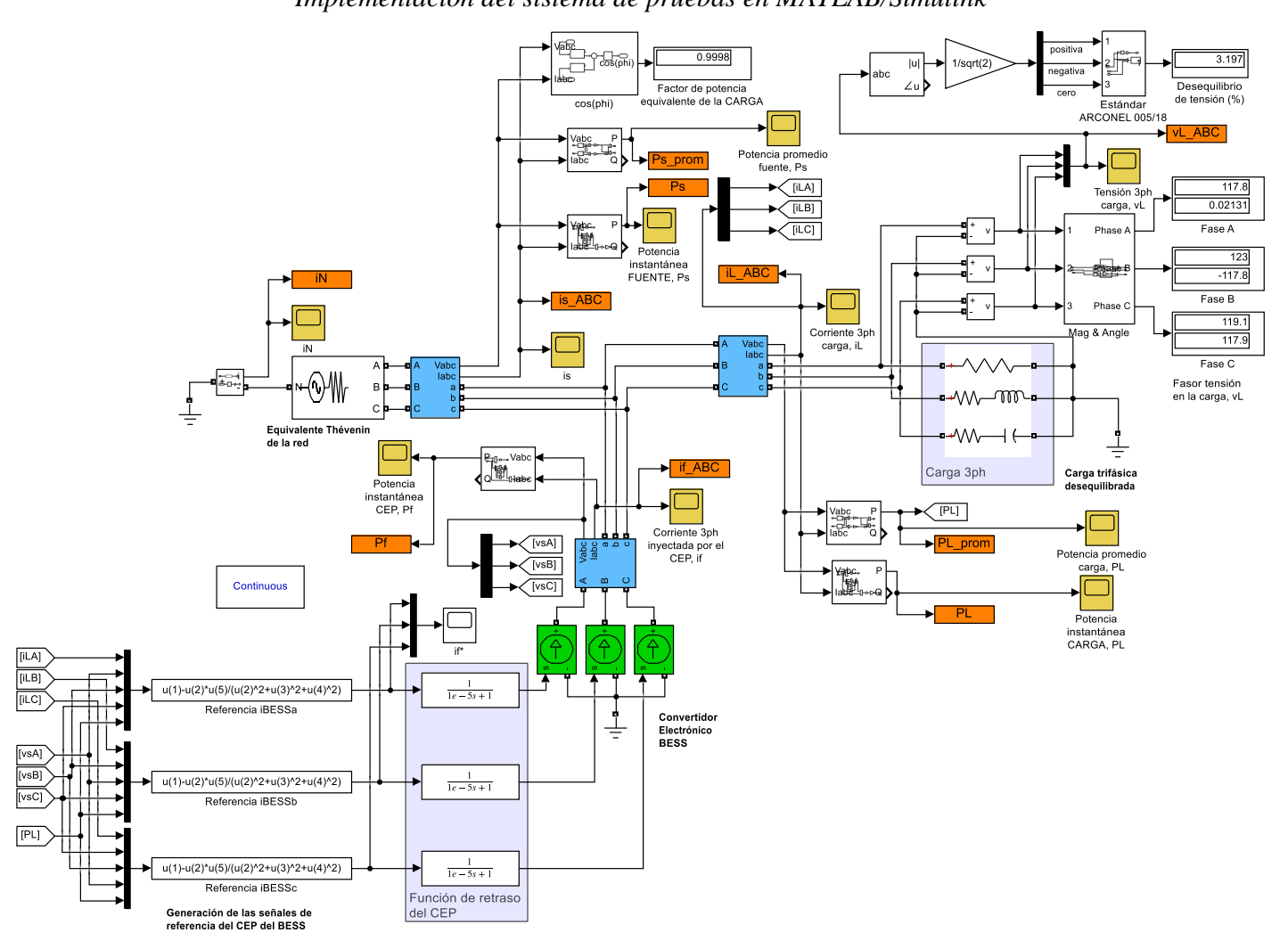

Fuente: Elaboración propia 
Caso base

En primer lugar se estudia el caso base, en el cual el BESS no interviene en las labores de compensación del desequilibrio de la carga, sino la red eléctrica principal, quien alimenta a la carga en su totalidad. Este caso puede ser visto también desde otro enfoque: que el BESS se encuentre entregando o absorbiendo potencia de un modo convencional, es decir, mediante la generación de corrientes trifásicas equilibradas, de tal manera que la única fuente de desequilibrio sea la carga trifásica. La Figura 9 muestra los resultados obtenidos en la simulación del caso base. La Figura 9(a) ilustra la dinámica adoptada por tensión fase-neutro en el PCC para el horizonte temporal considerado. Si bien, en esta ilustración apenas se aprecia el desequilibrio de tensión, al echar un vistazo a los valores de las componentes de secuencia y aplicar la ecuación (1) se puede verificar que el desequilibrio asciende al $3.20 \%$, valor que se sitúa por encima de lo que exige el código de red ecuatoriano. El desequilibrio se hace más evidente si se observa la corriente de carga instantánea, $i_{L}$, en la Figura 9(b). En este caso, la corriente de la red principal (Figura 9(d)) adopta la misma forma que la de carga, por cuanto se ha forzado al BESS a no inyectar ni absorber potencia en el PCC (Figura 9(c)).

A manera de comprobación, en la Figura 10(a) se ha graficado el lugar geométrico descrito por las componentes simétricas instantáneas de la corriente inyectada por la red principal en el PCC, obtenido al muestrear $i_{s}$ (Figura 9(d)), dentro la ventana 0.05s-0.1s, y evaluar la ecuación (2), expresada en términos de la corriente. El lugar geométrico refleja la falta de correlación entre las componentes de secuencia positiva y negativa, brindando un diagnóstico visual del desequilibrio. Por complementariedad, en la Figura 10(b) se muestra la dinámica de la corriente medida en el neutro de la microrred, cuya amplitud da testimonio de la severidad del desequilibrio estudiado.

Figura 9

Resultados de la simulación (caso base)

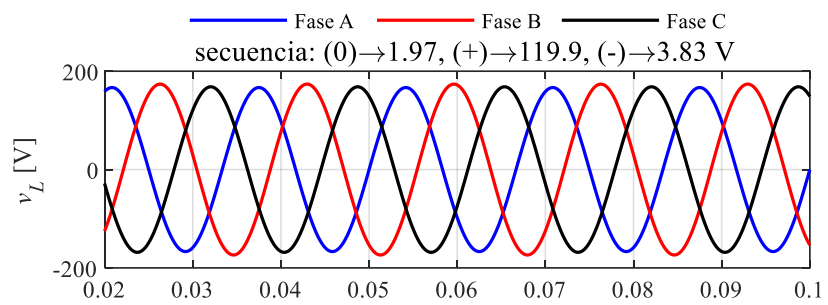

(a)

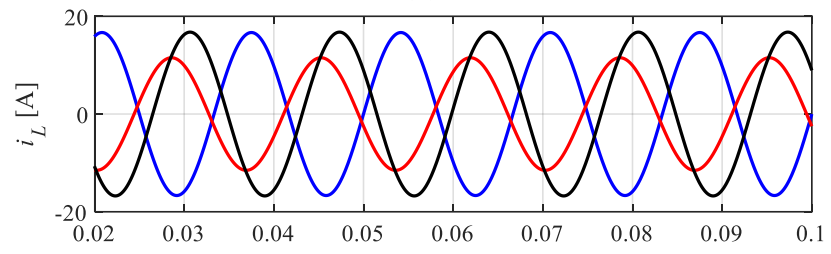

(b)

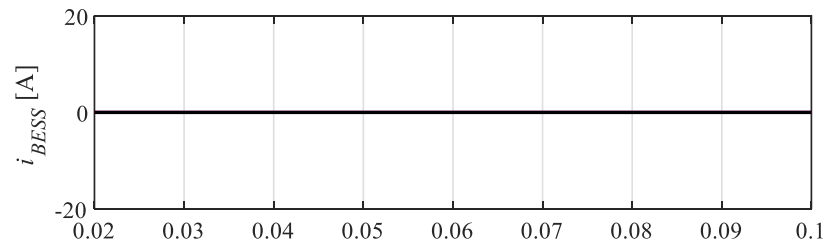

(c)

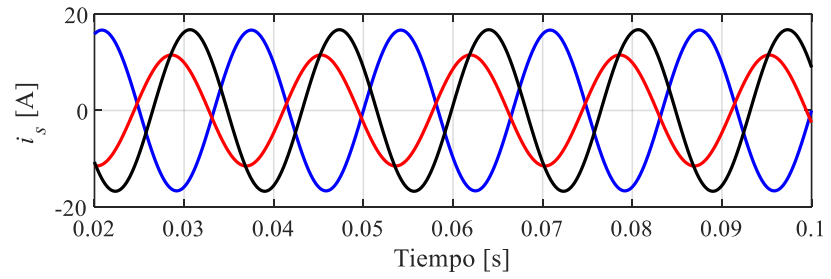

(d)

Fuente: Elaboración propia 


\section{Figura 10}

Resultados de la simulación (caso base): a) lugar geométrico componentes simétricas instantáneas (is, red principal); b) corriente instantánea del neutro, $i_{N}$

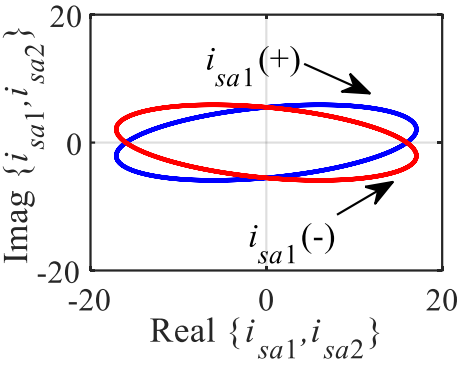

(a)

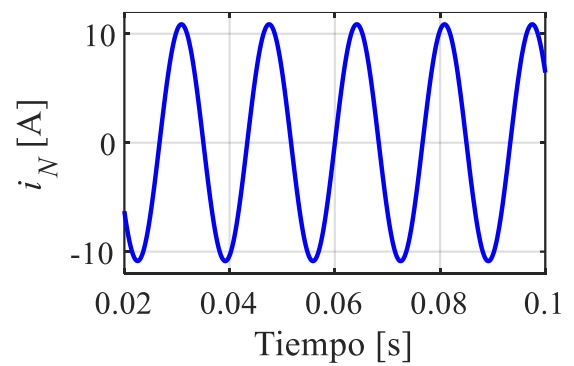

(b)

Fuente: Elaboración propia

Por último, en la Figura 13 se presenta la dinámica adoptada por la potencia instantánea y promedio inyectada/absorbida en el PCC por cada uno de los agentes del sistema de prueba, antes y después de la compensación.

\section{Participación del BESS en la mitigación del desequilibrio}

Para este estudio, se procede a habilitar el esquema de compensación implementado en el CEP del BESS y se repite el experimento descrito en el caso base. Con el propósito de hacer una primera verificación de la efectividad de la propuesta, se simularán condiciones operativas del CEP prácticamente ideales, al considerar, por ejemplo, una constante de tiempo $T_{C}=1 \times 10^{-5} \mathrm{~s}$ en la función de retardo (Figura 7). Además, para la evaluación del conjunto de ecuaciones (14)-(16) se considera un valor de $k=0$, con lo que se podrá obtener un factor de potencia unitario con la actuación del CEP bajo este modo operativo.

La Figura 11(a) ilustra la dinámica de tensión fase-neutro en el PCC. En esta ilustración se observa que el desequilibrio de tensión en la carga ha sido reducido sustancialmente, pues la componente de secuencia negativa ha disminuido su valor de forma apreciable. La aplicación de (1) con estos datos revela que el desequilibrio de tensión (según ARCONEL 005/18) se reduce al $0.18 \%$.

Si bien, la corriente instantánea en la carga permanece aún desequilibrada (Figura 11(b)), es la acción de control del BESS (Figura 11(c)) la que permite compensar la corriente de carga equivalente en el PCC. La Figura 11(d) muestra cómo esta corriente es equilibrada desde el punto de vista de la red principal (aguas arriba del PCC) y, además, cómo esta corriente está en fase con la tensión trifásica medida en ese punto $(\cos \varphi=1)$. Esto último, se debe a la asignación $k=0$ en el esquema de control del BESS propuesto.

\section{Figura 11}

Compensación del desequilibrio por parte del BESS

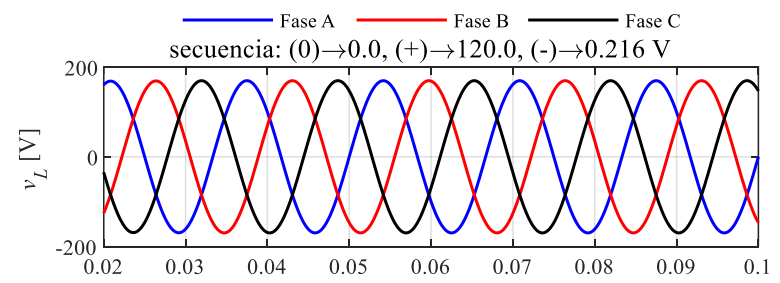

(a) 


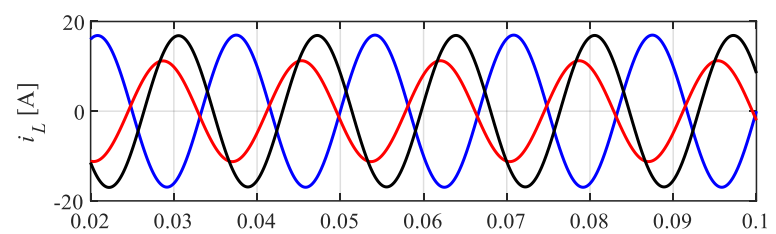

(b)

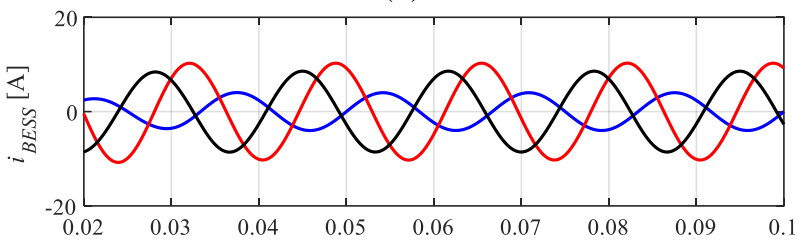

(c)

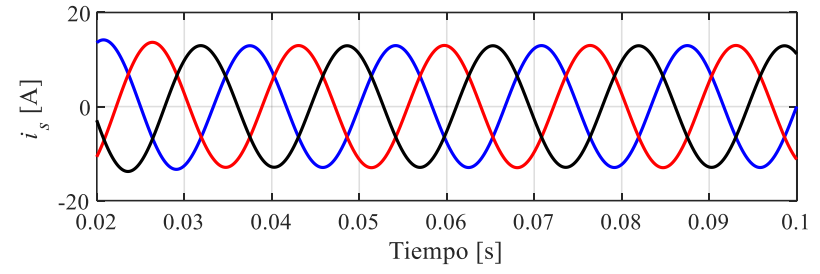

(d)

Fuente: Elaboración propia

Siguiendo un procedimiento similar a aquel descrito en el caso base, en la Figura 12(a) se ha trazado el lugar geométrico de las componentes simétricas instantáneas de la corriente suministrada por la fuente, en la cual se puede evidenciar la labor exitosa del BESS en la mitigación del desequilibrio; las curvas están solapadas. Este diagnóstico lo respalda la ausencia de una corriente circulante por el neutro de la microrred (Figura 12(b)) y permite verificar el cumplimiento del primer objetivo de control.

Figura 12

Compensación del desequilibrio por parte del BESS: a) lugar geométrico componentes simétricas instantáneas ( $i_{s}$, red principal); b) corriente instantánea del neutro, $i_{N}$

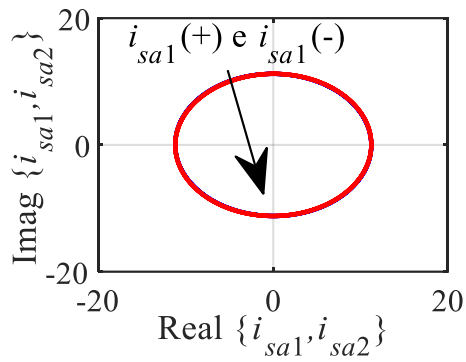

(a)

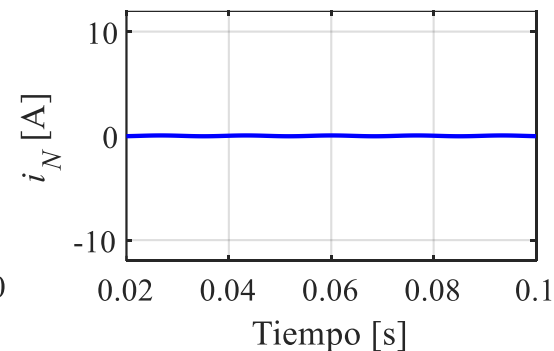

(b)

Fuente: Elaboración propia

En aras de la completitud, en la Figura 13 se presenta una comparación de las dinámicas de las potencias activas instantáneas manejadas por cada uno de los agentes energéticos del sistema de pruebas, antes y después de la intervención del BESS con sus labores de compensación. Nótese que, en el caso base (Figura 13(a)), la potencia instantánea ondulatoria demandada por la carga desequilibrada tiene que ser suplida completamente por la red principal. Si en este caso, el BESS inyectase/absorbiese potencia activa mediante el modo convencional, el resultado sería una línea continua en el tiempo que restaría/sumaría al aporte proveniente de la red principal para la cobertura de la potencia demandada. En todo caso, la generación de la componente ondulatoria de la potencia activa requerida por la carga seguiría siendo endosada a la red principal. Ahora bien, cuando se habilita la compensación del BESS en el sistema de prueba (ver Figura 13(b)), la componente oscilante de la potencia instantánea de la carga es asumida por el BESS, mientras que la red principal aporta únicamente la potencia promedio demandada por esta. Se cumple, por tanto, el segundo objetivo de control. 
Figura 13

Potencia activa instantánea: a) caso base, b) compensación por parte del BESS

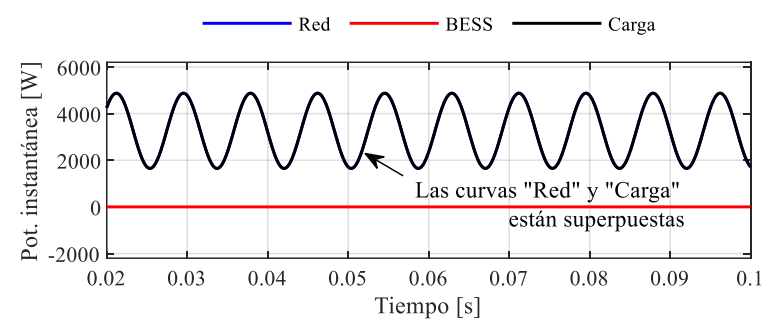

(a)

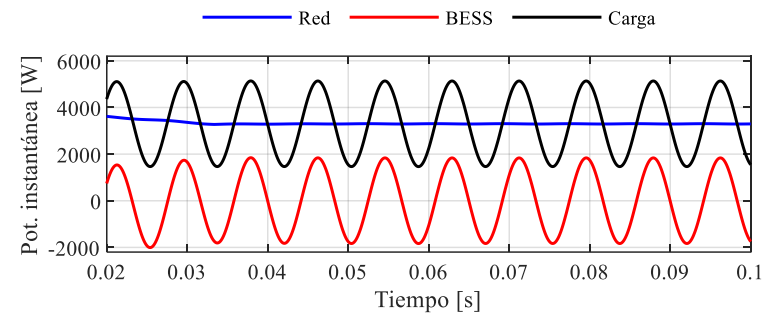

(b)

Fuente: Elaboración propia

Finalmente, la Tabla 1 ofrece los resultados más relevantes del estudio presentado.

Tabla 1

Resumen de los resultados

\begin{tabular}{|c|c|c|}
\hline & $\begin{array}{c}\text { Tensión fase-neutro medida } \\
\text { en el PCC [V] }\end{array}$ & $\begin{array}{c}\text { Desequilibrio } \\
\text { de tensión } \\
\text { (ARCONEL } \\
\text { 005/18) }\end{array}$ \\
\hline Caso base & $v_{L a}=117.8 \sqrt{2} \operatorname{sen}(\omega t)$ & \\
$v_{L b}=123.0 \sqrt{2} \operatorname{sen}\left(\omega t-117.8^{\circ}\right)$ & $3.196 \%$ \\
\hline $\begin{array}{c}\text { Compensación } \\
\text { mediante } \\
\text { BESS }\end{array}$ & $v_{L c}=119.1 \sqrt{2} \operatorname{sen}\left(\omega t+117.9^{\circ}\right)$ & \\
\hline & $v_{L b}=119.8 \sqrt{2} \operatorname{sen}(\omega t)$ & $0.180 \%$ \\
\hline
\end{tabular}

Fuente: Elaboración propia

\section{Conclusiones}

Entre los principales desafíos operativos derivados de la implementación de microrredes eléctricas (en modo isla) y sistemas eléctricos débiles y aislados, en general, se encuentran aquellos relacionados con el desequilibrio de tensiones y corrientes provocado por la naturaleza asimétrica de la carga trifásica en su operación en tiempo real. Según la severidad del desequilibrio, esta condición podría comprometer el correcto funcionamiento de los distintos componentes del sistema, reducir la eficiencia y su vida útil, y degradar los índices de calidad del suministro eléctrico. Aprovechando la proliferación de agentes energéticos con interfaz de conexión a red basado en convertidores electrónicos de potencia en una microrred eléctrica, como es el caso de los sistemas de almacenamiento energético en baterías (BESS), en este trabajo se ha propuesto una estrategia de control aplicada a tales convertidores con el objeto de mitigar el desequilibrio de tensión y/o corriente. La propuesta se encuentra sustentada en la teoría de las componentes simétricas instantáneas y la formulación resultante carece de complejidad matemática, lo que facilita su implementación práctica en los esquemas de control convencionales implementados en la electrónica del BESS. La efectividad brindada por la propuesta ha sido evaluada sobre un sistema eléctrico de pruebas, diseñado para los intereses de esta investigación, en el entorno de simulación MATLAB/Simulink. Los resultados obtenidos muestran que la incorporación de la estrategia de compensación en el convertidor electrónico de potencia del BESS 
permite reducir efectivamente el grado de desequilibrio en la microrred, para lo cual se ha tomado como referencia el código de red ecuatoriano. Estos resultados, además, han permitido corroborar los objetivos de control establecidos en el planteamiento de la propuesta y, con esto, verificar el cumplimiento de la hipótesis. Finalmente, es importante indicar que el alcance del estudio presentado en este artículo ha sido definido para presentar, de forma exhaustiva, la base teórica de la propuesta y brindar al lector una noción general de la interacción de los distintos actores de una microrred eléctrica, por lo que ha sido necesario realizar una serie de simplificaciones en la representación de sus modelos. A partir de esta base, se recomienda realizar simulaciones más detalladas en la que se consideren algunos aspectos, como el empleo de un modelo completo de los convertidores electrónicos de potencia; se tomen en cuenta las características de la batería y de su estado de carga; se considere un modelo desagregado de la microrred y que se incluyan los retardos asociados a las labores de medición, cómputo y procesamiento en el convertidor electrónico del BESS, los cuales podrían penalizar en mayor o menor medida la efectividad del método.

\section{Parámetros asignados al modelo de microrred:}

\section{Anexos}

Red eléctrica principal (fuente trifásica): $V_{a b(r m s)}=220 \mathrm{~V}, f=60 \mathrm{~Hz}$ y $z_{\text {th }}=0.77 \Omega$.

Carga desequilibrada (modelo serie): $R_{L A}=10.00 \Omega, R_{L B}=9.64 \Omega, L_{L B}=30.5 \mathrm{mH}$, $R_{L C}=8.66 \Omega$ y $C_{L C}=530.52 \mu \mathrm{F}$.

\section{Reconocimientos}

El autor expresa su agradecimiento a la Secretaría de Educación Superior, Ciencia, Tecnología e Innovación (SENESCYT) de la República del Ecuador, que, gracias a su apoyo financiero ha hecho posible su formación a nivel doctoral.

\section{Referencias}

Adouni, A., \& J. Marques Cardoso, A. (2021). Thermal Analysis of Low-Power Three-Phase Induction Motors Operating under Voltage Unbalance and Inter-Turn Short Circuit Faults. Machines, 9(1), 2. https://doi.org/10.3390/machines9010002

Agencia de Regulación y Control de Electricidad-ARCONEL. (2018a). Plan Maestro de Electricidad 2018-2027. https://www.recursosyenergia.gob.ec/plan-maestro-de-electricidad/

Agencia de Regulación y Control de Electricidad-ARCONEL. (2018b). Regulación No. ARCONEL 005/18. https://www.regulacionelectrica.gob.ec/wp-content/uploads/downloads/2019/01/Regulacion-deCalidad-Suscrita.pdf

Antić, T., Capuder, T., \& Bolfek, M. (2021). A Comprehensive Analysis of the Voltage Unbalance Factor in PV and EV Rich Non-Synthetic Low Voltage Distribution Networks. Energies, 14(1), 117. https://doi.org/10.3390/en14010117

Etxegarai, A., Eguia, P., Torres, E., Iturregi, A., \& Valverde, V. (2015). Review of grid connection requirements for generation assets in weak power grids. Renewable and Sustainable Energy Reviews, 41, 1501-1514. https://doi.org/10.1016/j.rser.2014.09.030

Fortescue, C. L. (1918). Method of Symmetrical Co-Ordinates Applied to the Solution of Polyphase Networks. Transactions of the American Institute of Electrical Engineers, XXXVII (2), 1027-1140. https://doi.org/10.1109/T-AIEE.1918.4765570

Ghosh, A., \& Joshi, A. (2000). The use of instantaneous symmetrical components for balancing a delta connected load and power factor correction. Electric Power Systems Research, 54(1), 67-74. https://doi.org/10.1016/S0378-7796(99)00071-1

Grainger, J. J., \& Stevenson, W. D. (2004). Análisis de sistemas de potencia. MacGraw-Hill. 
Gundumalla, V. B. K., \& Eswararao, S. (2018). Ramp Rate Control Strategy for an Islanded DC Microgrid with Hybrid Energy Storage System. 2018 4th International Conference on Electrical Energy Systems (ICEES), 82-87. https://doi.org/10.1109/ICEES.2018.8442363

Huang, S., Pham, D. C., Huang, K., \& Cheng, S. (2012). Space vector PWM techniques for current and voltage source converters: A short review. 2012 15th International Conference on Electrical Machines and Systems (ICEMS), 1-6.

Karimi, M., Farshad, M., Hong, Q., Laaksonen, H., \& Kauhaniemi, K. (2021). An Islanding Detection Technique for Inverter-Based Distributed Generation in Microgrids. Energies, 14(1), 130. https://doi.org/10.3390/en14010130

Ko, Y., \& Choi, W. (2021). A New SOC Estimation for LFP Batteries: Application in a 10 Ah Cell (HW 38120 L/S) as a Hysteresis Case Study. Electronics, 10(6), 705. https://doi.org/10.3390/electronics10060705

Kumar, G. V. B., \& Palanisamy, K. (2020). A Review of Energy Storage Participation for Ancillary Services in a Microgrid Environment. Inventions, 5(4), 63. https://doi.org/10.3390/inventions5040063

Kumar, T. P., Chandrashekar, Y., Subrahmanyam, N., \& Sydulu, M. (2015). Control strategies of a fuzzy controlled grid connected hybrid PV/PEMFC/battery distributed generation system. 2015 IEEE Power and Energy Conference at Illinois (PECI), 1-6. https://doi.org/10.1109/PECI.2015.7064932

Kundur, P. (1994). Power System Stability and Control (1st ed.). McGraw-Hill.

Li, D., Li, F., Rong, D., Zheng, K., Wang, D., \& Li, Q. (2018). An SVPWM Strategy for Multifunction Current Source Converter. 2018 IEEE International Power Electronics and Application Conference and Exposition (PEAC), 1-6. https://doi.org/10.1109/PEAC.2018.8590656

Nájera, J., Mendonça, H., de Castro, R. M., \& Arribas, J. R. (2019). Strategies Comparison for Voltage Unbalance Mitigation in LV Distribution Networks Using EV Chargers. Electronics, 8(3), 289. https://doi.org/10.3390/electronics8030289

Radwan, A., Khouri, I., \& Jiang, X. (2020). Modeling and Control of Current-Source Converter-Based AC Microgrids. 2020 IEEE 8th International Conference on Smart Energy Grid Engineering (SEGE), $97-$ 101. https://doi.org/10.1109/SEGE49949.2020.9181969

Saponara, S., Saletti, R., \& Mihet-Popa, L. (2019). Hybrid Micro-Grids Exploiting Renewables Sources, Battery Energy Storages, and Bi-Directional Converters. Applied Sciences, 9(22), 49-73. https://doi.org/10.3390/app9224973 